\title{
Role of I-123 MIBG in sepsis-induced cardiomyopathy
}

\author{
Fahad Waqar, MD, ${ }^{\mathrm{a}}$ Hina K. Jamali, $M D{ }^{\mathrm{a}}$ and Myron C. Gerson, $\mathrm{MD}^{\mathrm{a}}$ \\ a Division of Cardiovascular Health and Disease, Department of Internal Medicine, \\ University of Cincinnati College of Medicine, Cincinnati, Ohio
}

Received Aug 22, 2016; accepted Aug 22, 2016

doi:10.1007/s12350-016-0659-0

\section{See related article, pp. 483-491}

\section{SEPSIS AND CARDIAC DYSFUNCTION}

Incidence of cardiac dysfunction, both right ventricular (RV) and left ventricular (LV), as well as systolic and diastolic, is high among patients with sepsis and septic shock. ${ }^{1}$ The term "septic cardiomyopathy" is often used to describe the spectrum of cardiac dysfunction associated with clinical effects of sepsis. Although commonly thought to be reversible in most survivors, ${ }^{2}$ cardiac dysfunction is associated with overall worse outcome ${ }^{3}$ and mortality. The study by Landesberg and colleagues is one of the largest and most important human studies on this subject. For patients with severe sepsis and septic shock, those authors showed that both LV systolic and diastolic dysfunction are associated with significantly higher mortality when present. In experimental animal models of sepsis and in the relatively small available human studies that have explored the pathophysiology of cardiac dysfunction in sepsis, several associated and, likely, causative mechanisms have been described. Among these, notable mechanisms include alterations and dysfunction of cardiac calcium channels, ${ }^{4}$ cardiomyocyte damage caused by excessive nitrous oxide, ${ }^{5}$ cytokines including IL-1, TNF-alpha and IL- $6,{ }^{1}$ oxidative

Funding Funded in part by the John R. Strauss Fund for Research and Education in Cardiovascular Imaging.

Reprint requests: Fahad Waqar, MD, Division of Cardiovascular Health and Disease, Department of Internal Medicine, University of Cincinnati College of Medicine, P.O. Box 670542, Cincinnati, OH, 45267-0542; fahad.waqar@uc.edu

J Nucl Cardiol 2018;25:492-5.

$1071-3581 / \$ 34.00$

Copyright (c) 2016 American Society of Nuclear Cardiology. stress $^{6}$ and, to some extent, microvascular myocardial ischemia. ${ }^{7}$ However, it is worth emphasizing that unlike earlier hypotheses had suggested, decrease in coronary perfusion is usually not seen in sepsis and in fact, there might be an increase in global coronary perfusion. ${ }^{8}$

Methodology suggested in the literature regarding diagnosis of myocardial dysfunction in sepsis is inconsistent. ${ }^{1}$ Although traditionally ejection fraction is a commonly used measure of LV systolic dysfunction, its relationship with and influence by hemodynamic changes involving preload and afterload in sepsis is complex. This makes LV ejection fraction (LVEF) an unreliable measure of myocardial performance in septic patients. ${ }^{9}$ LVEF alone precludes assessment of diastolic and RV dysfunction, which are commonly impaired in patients with sepsis. ${ }^{3,10}$ These limitations have led to more common use of echocardiography with tissue Doppler technique, ${ }^{11}$ contrast echocardiography, ${ }^{12}$ and strain imaging. ${ }^{13}$ These newer techniques, although extensively studied and showing substantial promise for non-septic conditions, lack strong documentation of their effectiveness for evaluation of sepsis. Importantly, current guidelines focus directly on the therapeutic approach to treatment of sepsis rather than on prevention or treatment of the component of ventricular dysfunction in these patients. The present study focuses attention on the cardiac autonomic nervous system, to investigate the occurrence of autonomic dysfunction in the development of cardiac dysfunction in sepsis. Improved understanding of the role of cardiac autonomic dysfunction in the pathophysiology of sepsis may lead to new methods to mitigate the development of ventricular dysfunction and improve outcomes.

\section{AUTONOMIC DYSFUNCTION AND SEPSIS}

Exaggerated sympathetic stimulation is a well-established "fight and flight" mechanism during any stress, and sepsis is no exception. Adrenergic "overshoot" can often arise in critical illnesses and sepsis, resulting in serious detrimental effects beyond any protective effects. ${ }^{14}$ During 
sepsis, there is a decrease in the re-uptake of the neurotransmitters epinephrine and norepinephrine from the synaptic gap into the presynaptic nerve endings and increase in their secretion into the circulation. ${ }^{15}$ This leads to a much slower systemic degradation and much higher serum levels of these neurotransmitters ${ }^{16}$ and is predictive of outcome. ${ }^{17,18}$ This effect is further compounded by exogenous use of catecholamines commonly seen in practice for treatment of septic shock patients. ${ }^{19}$ High cytokine production during sepsis also leads to down-regulation of adrenergic receptors $^{20}$ adding to overall autonomic dysfunction. Among several vital organs, the heart is particularly influenced by sympathetic stimulation and overstimulation during sepsis. This is often evident by tachycardia, inotropy, and lusitropy. In addition to being an important protective mechanism, these effects are thought to be the major source of diastolic dysfunction seen in patients with sepsis and septic shock. ${ }^{21}$ Several pathophysiologic mechanisms of systolic dysfunction and direct myocardial damage mentioned above are also thought to directly result from high catecholamine levels in the circulation. ${ }^{22}$

\section{MIBG IMAGING IN CARDIAC DYSFUNCTION}

The catecholamine analog, ${ }^{123}$ I-metaiodobenzylguanidine (MIBG), provides a noninvasive measure of cardiac sympathetic neural activity. Several studies have demonstrated the role of ${ }^{123}$ I-MIBG in predicting outcome in heart failure patients. Most notable among these is the AdreView Myocardial Imaging for Risk Evaluation in Heart Failure (ADMIRE-HF) trial. ${ }^{23}$ This prospective study concluded that a heart-to-mediastinum uptake ratio of $\geq 1.6$ was associated with significantly better outcome among heart failure patients. Several other studies have shown similar results. ${ }^{123} \mathrm{I}-\mathrm{MIBG}$ based imaging is also shown to be helpful in guiding medical and device-based therapy among heart failure patients. ${ }^{24}$ Despite extensive, almost all of the literature on utility of ${ }^{123}$ I-MIBG-based imaging are gathered from patients with chronic systolic heart failure. Relatively few data for ${ }^{123}$ I-MIBG imaging are available for patients with isolated diastolic dysfunction or acute illness-related cardiomyopathy such as septic cardiomyopathy. ${ }^{25,26}$

\section{MIBG IMAGING AND SEPSIS: PRESENT STUDY}

The present novel and well-designed study addresses the usefulness of ${ }^{123}$ I-MIBG imaging in the relatively common but pathophysiologically distinct entity of sepsisinduced cardiomyopathy. Cardiac systolic and diastolic function are assessed by conventional echocardiographic/ Doppler techniques in rodents treated with lipopolysaccharide to induce a sepsis syndrome. MIBG SPECT cardiac adrenergic imaging is then used in a separate group of lipopolysaccharide-administered animals and compared to the results in controls. The results are impressive, consistently suggesting significant difference between control animals and those with lipopolysaccharide-induced sepsis. One limitation, however, is that one group of sepsisinduced rats underwent MIBG imaging and a different group had echocardiography/Doppler assessment of myocardial function. Although both groups showed significant differences when compared to controls, imaging of ventricular function and neural imaging will likely need to be tested in the same group of subjects to prove that autonomic changes seen with ${ }^{123}$ I-MIBG imaging correlate with abnormalities in myocardial function tested by conventional echocardiography/Doppler methods. Second, although echocardiographic techniques used to assess systolic function are robust, parameters used to evaluate diastolic function in the present study are limited (E/A ratio only). In addition, diastolic dysfunction is frequently seen in patients with systolic dysfunction and can result in an abnormal E/A ratio. ${ }^{27}$ Results of the present study regarding diastolic dysfunction among lipopolysaccharidetreated animals are difficult to interpret without testing animals with pure diastolic dysfunction.

There are several pathophysiologic differences between cardiomyopathies leading to chronic heart failure compared to acute cardiomyopathy induced by sepsis, most important being prolonged overstimulation of post-synaptic adrenergic receptors leading to catabolism and down-regulation of these receptors in chronic cases of heart failure. This down-regulation of receptors forms an important basis for washout rate measured as part of MIBG-based imaging. Although, theoretically, similar washout effects should be seen with increased presynaptic levels of catecholamines due to overall high levels of catecholamines in the circulation (endogenous and exogenous), few studies have been done so far among animal models or human subjects with acute illness (e.g., sepsis). In addition, it is yet to be studied how MIBG-based imaging can help in predicting outcome in a population of patients with sepsis since exogenous catecholamines in this population offer additional competition for norepinephrine analogs such as ${ }^{123} \mathrm{I}-\mathrm{MIBG}$ for receptor binding. Also, sympathetic overstimulation in chronic heart failure may be a phenomenon relatively specific to the heart while sympathetic overstimulation in sepsis affects a wide range of other vital organs as well. This will be an important point to keep in mind when using the heart-tomediastinum ratio for which an assumption is required that "background" activity represented by the mediastinal region of interest is not altered by changes in blood flow to other organs. It may be necessary to calculate absolute uptake of ${ }^{123}$ I-MIBG in the heart rather than only measure heart activity relative to the 
mediastinum or other extra-cardiac structures. Lastly, it is well known that the uptake-1 mechanism of norepinephrine re-uptake is the predominant mechanism in humans while in rodents a much higher proportion of uptake- 2 mechanism is seen. The authors have explained that this difference likely only strengthens the results of the study and the higher uptake-1 mechanism in humans will make the differences observed among cases and control even more prominent. While this may be true, this can only be proved once tested in human subjects.

\section{FUTURE DIRECTIONS}

Critical illness-associated cardiomyopathy, especially sepsis-induced cardiac dysfunction, is an extremely common phenomenon with poor outcome. Extensive studies have been done to understand the underlying mechanisms over the past 6 decades but little is known regarding what can be done to predict outcome and prevent adverse events in this population. To date, most sepsis guidelines focus on direct treatment of infection and associated hemodynamic problems. Myocardial dysfunction is currently seen as a complication of sepsis and sepsis treatment. Some of the vital therapies used in septic patients, including fluid resuscitation and vasopressors, likely contribute to myocardial dysfunction and sympathetic overstimulation in a large proportion of these patients. Techniques like ${ }^{123}$ I-MIBG-based imaging and more sophisticated echocardiographic techniques have the potential to help clinicians carefully select patients at higher risk of developing cardiac dysfunction and tailor their vasopressor and inotropic therapy accordingly. Although there are no randomized trials on this subject, several small studies suggest a controversial but potentially beneficial role of adrenergic blocker therapies in septic patients. ${ }^{28,29}$ The role of adrenergic overstimulation in development of myocardial dysfunction in septic patients and the potential role of autonomic modulation in its treatment and prevention require further investigation.

\section{Disclosure}

None.

\section{References}

1. Antonucci E, Fiaccadori E, Donadello K, Taccone FS, Franchi F, Scolletta S. Myocardial depression in sepsis: From pathogenesis to clinical manifestations and treatment. J Crit Care. 2014; 29(4):500-11.

2. Vieillard-Baron A. Septic cardiomyopathy. Ann Intensive Care. 2011;1(1):6.
3. Landesberg G, Gilon D, Meroz Y, Georgieva M, Levin PD, Goodman S, et al. Diastolic dysfunction and mortality in severe sepsis and septic shock. Eur Heart J. 2012;33(7):895-903.

4. Stengl M, Bartak F, Sykora R, Chvojka J, Benes J, Krouzecky A, et al. Reduced L-type calcium current in ventricular myocytes from pigs with hyperdynamic septic shock. Crit Care Med. 2010;38(2):579-87.

5. Kirkeboen KA, Strand OA. The role of nitric oxide in sepsis: An overview. Acta Anaesthesiol Scand. 1999;43:275-88.

6. Galley FH. Oxidative stress and mitochondrial dysfunction in sepsis. Br J Anaesth. 2011;107(1):57-64.

7. Groeneveld $A B$, van Lambalgen AA, van den Bos GC, Bronsveld W, Nauta JJ, Thijs LG. Maldistribution of heterogeneous coronary blood flow during canine endotoxin shock. Cardiovasc Res. 1991;25(1):80-8.

8. Cunnion RE, Schaer GL, Parker MM, Natanson C, Parrillo JE. The coronary circulation in human septic shock. Circulation. 1986;73(4):637-44.

9. Repessé X, Charron C, Vieillard-Baron A. Evaluation of left ventricular systolic function revisited in septic shock. Crit Care. 2013;17(4):164.

10. Vincent JL, Reuse C, Frank N, Contempré B, Kahn RJ. Right ventricular dysfunction in septic shock: Assessment by measurements using the thermodilution technique. Acta Anaesthesiol Scand. 1989;33(1):34-8.

11. Weng L, Liu YT, Du B, Zhou JF, Guo XX, Peng JM, et al. The prognostic value of left ventricular systolic function measured by tissue Doppler imaging in septic shock. Crit Care. 2012;16(3):R71.

12. Sado D, Greaves K. Myocardial perfusion echocardiography: A novel use in the diagnosis of sepsis-induced left ventricular systolic impairment on the intensive care unit. Eur $\mathrm{J}$ Echocardiogr. 2011;12(1):81-4.

13. Chu M, Gao Y, Zhou B, Wu B, Wang J, Xu D. Circumferential strain can be used to detect lipopolysaccharide-induced myocardial dysfunction and predict the mortality of severe sepsis in mice. PLoS One. 2016;11(5):e0155346.

14. Dünser MW, Hasibeder WR. Sympathetic overstimulation during critical illness: Adverse effects of adrenergic stress. J Intensive Care Med. 2009;24(5):293-316.

15. Guyton AC, Hall JE. The autonomic nervous system and the adrenal medulla. In: Guyton AC, Hall JE, editors. Textbook of medical physiology. Philadelphia, PA: W.B. Saunders; 2000. p. 697-708.

16. Jones SB, Romano FD. Dose- and time-dependent changes in plasma catecholamines in response to endotoxin in conscious rats. Circ Shock. 1989;28(1):59-68.

17. Boldt J, Menges T, Kuhn D, Diridis C, Hempelmann G. Alterations in circulating vasoactive substances in the critically ill: A comparison between survivors and non-survivors. Intensive Care Med. 1995;21(3):218-25.

18. Benedict CR, Rose JA. Arterial norepinephrine changes in patients with septic shock. Circ Shock. 1992;38(3):165-72.

19. Annane D, Bellissant E, Cavaillon JM. Septic shock. Lancet. 2005;365(9453):63-78.

20. Bucher M, Kees F, Taeger K, Kurtz A. Cytokines down-regulate alpha1-adrenergic receptor expression during endotoxemia. Crit Care Med. 2003;31(2):566-71

21. Bouhemad B, Nicolas-Robin A, Arbelot C, Arthaud M, Féger F, Rouby JJ. Isolated and reversible impairment of ventricular relaxation in patients with septic shock. Crit Care Med. 2008;36(3):766-74.

22. Iwai-Kanai E, Hasegawa K, Araki M, Kakita T, Morimoto T, Sasayama S. Alpha- and beta-adrenergic pathways differentially regulate cell type-specific apoptosis in rat cardiac myocytes. Circulation. 1999;100(3):305-11. 
23. Jacobson AF, Senior R, Cerqueira MD, Wong ND, Thomas GS, Lopez VA, et al. Myocardial iodine-123 meta-iodobenzylguanidine imaging and cardiac events in heart failure. Results of the prospective ADMIRE-HF (AdreView Myocardial Imaging for Risk Evaluation in Heart Failure) study. J Am Coll Cardiol. 2010;55(20):2212-21.

24. Waqar F, Dunlap SH, Gerson MC. What will be the role of I-123 MIBG in improving the outcome of medically treated heart failure patients? J Nucl Cardiol. 2012;19(6):1198-205.

25. Sugiura M, Yamamoto K, Takeda Y, Takeda Y, Dohmori T, Ogata $\mathrm{M}$, et al. The relationship between variables of 123-Imetaiodobenzylguanidine cardiac imaging and clinical status of the patients with diastolic heart failure. Int $\mathrm{J}$ Cardiol. 2006;113(2):223-8.
26. Verschure DO, van Eck-Smit BL, Somsen GA, Verberne HJ. Cardiac sympathetic activity in hypertrophic cardiomyopathy and Takotsubo cardiomyopathy. Clin Transl Imaging. 2015;3(5):379-85.

27. Zile MR, Brutsaert DL. New concepts in diastolic dysfunction and diastolic heart failure: Part I: Diagnosis, prognosis, and measurements of diastolic function. Circulation. 2002;105(11): 1387-93.

28. Chacko CJ, Gopal S. Systematic review of use of $\beta$-blockers in sepsis. J Anaesthesiol Clin Pharmacol. 2015;31(4):460-5.

29. de Montmollin E, Aboab J, Mansart A, Annane D. Bench-tobedside review: Beta-adrenergic modulation in sepsis. Crit Care. 2009;13(5):230. 